\title{
Concepciones diferentes del término raíz: aportes de la experiencia vivencial del trabajo de campo
}

\author{
Conceptions different from the termination root: contribution of the vivential \\ experience of field work
}

Antonio Vera

ajvera68@gmail.com

Código ORCID: 0000-0002-8298-9750

Universidad del Zulia, Venezuela
Maritza Martínez

mmartinez.luz@gmail.com

Código ORCID: 0000-0001-5914-5878

Universidad del Zulia, Venezuela

Articulo recibido en enero 2019 Arbitrado en febrero 2019 Publicado en julio 2019

Resumen

Se determinaron concepciones diferentes del término raíz durante la experiencia vivencial del trabajo de campo. La metodología fue descriptiva, de campo y no experimental. Los estudiantes "descubrieron" raíces con rasgos anatómicos, morfológicos, ecológicos y fisiológicos diferentes en comparación al concepto convencional. Se identificaron raíces haustorios (hemiparásitas); para la fijación físicamecánica a sustratos (epífitas); engrosadas por acumulación de sustancias de reserva (arbusto); fúlcreas para el "anclaje" y la ultrafiltración de sales (mangle rojo) y neumatóforos con geotropismo negativo para absorber oxígeno atmosférico (mangles blanco y negro). Los estudiantes obtuvieron alcances como focalización de la atención a situaciones problema, consolidación de analisis y conclusiones, analisis de nuevas interrelaciones cognitivas, enriquecimiento del conocimiento disciplinar y lenguaje científico, aplicación de procesos mentales y método científico, afianzamiento de interacciones ecológicas y adaptaciones, comparación y analisis de nuevas concepciones con el concepto tradicional de raíz y socialización del conocimiento. Las nuevas concepciones podrían generar cambios conceptuales en los estudiantes.

Palabras clave: Cambio conceptual; Botánica; ideas previas; trabajo de campo; concepto de raíz

\begin{abstract}
Different conceptions of the root term were determinate during the experience of fieldwork. The methodology was descriptive, field and non-experimental. The students "discovered" roots with different anatomical, morphological, ecological and physiological features compared to the conventional concept. Haustorium roots (hemiparasites), roots for the physical-mechanical fixation to substrates (epiphytes), thickened roots by the accumulation of reserve substances (shrub), prop roots for anchoring and ultrafiltration of salts (red mangrove) and pneumatophores roots with negative geotropism to absorb atmospheric oxygen (white mangrove and black mangrove) were identified. Students achieved scopes like focusing of attention on problem situations, consolidation of analysis and conclusions, analysis of new cognitive interrelations, enrichment of disciplinary knowledge and scientific language, strengthening of ecological interactions and adaptations, comparison and analysis of new conceptions with the traditional concept of root and socialization of the knowledge. The new conceptions could to generate conceptual changes in students.
\end{abstract}

Keywords: Conceptual change; Botany; previous ideas fieldwork; root concept 


\section{INTRODUCCIÓN}

La naturaleza del conocimiento biológico se centra en su carácter empírico, (estudio de las diversas manifestaciones de la vida), los datos fácticos y el campo de lo observacional para la concreción de problemas de investigación y el establecimiento de teorías biológicas (Méndez Méndez y González, 2019).

Sin embargo, a pesar de estos rasgos inherentes al conocimiento biológico, que le ofrecen información basada en lo fáctico (la observación y los sentidos), es muy importante elevar el carácter didáctico de la enseñanza y el aprendizaje de la Biología a las construcciones conceptuales, al reconocimiento de su sistematicidad y a las implicaciones sociales de la ciencia (Padrón, 2016).

En función de lo expuesto, hoy en día el educador de Biología ha emitido un salto cualitativo en las actividades experimentales que ejecuta al impartir el conocimiento biológico, cambiando la utilización de las prácticas de laboratorio por los trabajos de campo, las expediciones e incluso realizando visitas por los mismos espacios (entornos) de la propia unidad educativa donde desempeña su rol didáctico. Todo esto con el objeto de promover la expresión del pensamiento de los estudiantes, la comprensión del mundo y la interrelación con acontecimientos de la cotidianidad (Acevedo, 2004; Álvarez, 2007; Tacca, 2010).

En este sentido, el trabajo de campo brinda al docente la oportunidad de diversificar y viabilizar la enseñanza y el aprendizaje de la Biología de manera innovadora, enriquecedora e integral para el educando a través de distintas actividades, experiencias, desempeño de roles entre otros aspectos. Es por ello que el trabajo de campo se define como una estrategia didáctica planificada y valiosa en la que el docente le imprime una gran intencionalidad con objetivos trazados, a objeto que el estudiante tenga una experiencia práctica-directavivencial in situ y mantenga una relación en contacto con el objeto de aprendizaje en su propio ambiente natural.

De acuerdo a autores como Oviedo y Dávila (2001), el trabajo de campo permite a los estudiantes la "indagación a primera mano" y de esta manera aprenden a conocer su campo o entorno inmediato, así como a formular y responder preguntas.

Buitrago Suárez (2011) ha señalado que el trabajo de campo favorece el desarrollo de capacidades en los estudiantes para internalizar conceptos básicos, mientras que Vera y Martínez (2013) indicaron que esta estrategia didáctica permite en los educandos la adquisición de experiencias que enriquecen su aprendizaje vivencial ecológico.

De igual modo, se ha definido a la huerta escolar como un sistema ecológico que permite educar a los estudiantes en la comprensión de las interacciones y dinámicas ecológicas que ocurren en estos agroecosistemas, con miras a desarrollar una capacidad analítica que les permita extrapolar estos conocimientos a otros ámbitos de las ciencias naturales (Tobar et el., 2020).

El conocimiento de la Botánica no escapa a la acción didáctica que ejerce sobre sí mismo el trabajo de campo, y en particular la utilización de algunos espacios no convencionales para la praxis educativa como el supermercado para la enseñanza de la Taxonomía Botánica bajo un enfoque CTS (Ciencia, Tecnología y Sociedad) y apoyado en el constructivismo del aprendizaje (Moreno, 2007a); así como también se ha 
señalado al herbario como un ambiente didáctico para la enseñanza de la Biología Vegetal y otras áreas del conocimiento afines (Moreno, 2007b).

De tal manera que el campo, ambiente, espacio natural o ecosistema se emplea como marco de referencia para desarrollar, ejercitar, manejar y poner en práctica conocimientos, contenidos, temas y conceptos de la Botánica, destacando el área de la Organografía Vegetal, disciplina que comprende el estudio anatómico y morfológico de los órganos de las plantas superiores o vasculares como flor, fruto, hoja, tallo y raíz. (Parisca et al., 1985)

Por su parte, el cambio conceptual, justamente involucra (se centra) en la transformación, modificación o reajuste sobre el conocimiento que se tenía referente a algún asunto, contenido, materia, tema, término o vocablo.

El objetivo de esta investigación es determinar concepciones diferentes del término raíz a partir de la experiencia vivencial del trabajo de campo.

\section{MÉTODO}

El estudio se llevó a cabo siguiendo una metodología descriptiva, de campo y no experimental.

Las actividades practicas didácticas 0 trabajos de campo se llevaron a cabo en el bosque xerófilo y el bosque de manglar (en número de dos para cada uno de estos ecosistemas) de la Reserva de Fauna Silvestre Ciénaga de La Palmita e Isla de Pájaros, del Municipio Santa Rita del Estado Zulia, Venezuela en marzo de 2017 (I periodo académico) y septiembre de 2017 (II periodo académico).

Los estudiantes que formaron parte de la investigación correspondieron a alumnos cursantes de la cátedra de Botánica de la Mención Biología de la Escuela de Educación de la Facultad de Humanidades y Educación de la Universidad del Zulia. Por su parte, los autores del trabajo participaron como docentes mediadores durante el desarrollo de las prácticas de campo, e igualmente laboran en el dictado de la mencionada cátedra.

En la investigación se utilizaron dos grupos de estudiantes, uno para cada periodo académico de 2017, y éstos estuvieron formados por 18 alumnos e integrados en equipos de trabajo de tres participantes cada uno.

Los ambientes 0 espacios didácticos naturales fueron visitados y explorados por los docentes de forma previa a objeto de conocer los rasgos y potencialidades didácticas de la zona. También se trazó una ruta, a través de un sendero de 1,3 km, para realizar el recorrido y se realizaron paradas o estaciones definidas para el desarrollo de las diferentes actividades.

El desarrollo de las actividades prácticas se realizó a partir del trabajo de cada equipo de estudiantes el cual consistió en hacer observaciones, anotaciones, descripciones, discusiones intra e intergrupales, planteamientos de preguntas, inquietudes y dudas, uso de las ideas previas, formulación de hipótesis y recolectas de muestras botánicas. A nivel de las estaciones, los equipos de estudiantes formaron pequeñas sesiones plenarias para la exposición de ideas, confrontación de opiniones y generación de conclusiones.

La evaluación de las actividades prácticas de campo se complementó por medio de la entrega de un informe a cargo de cada grupo de alumnos. Igualmente los profesores tomaron nota de aspectos 
importantes suscitados en los trabajos de campo para la su calificación.

La investigación se llevó a cabo a través de una orientación metodológica de campo cuyos hallazgos se alcanzaron y tomaron de manera directa durante las prácticas didácticas realizadas en los ecosistemas de bosque xerófilo y manglar. Además el estudio fue no experimental ya que la variable investigada no fue manipulada.

\section{RESULTADOS}

En las experiencias vivenciales de los trabajos de campo, los estudiantes determinaron cinco (5) concepciones diferentes del término raíz (raíces almacenadoras de sustancias de reserva, raíces delgadas para fijación mecánica, raíces haustorios, raíces fúlcreas y raíces neumatóforos) Tabla 1, en comparación con el conocimiento biológico que poseían sobre la definición tradicional del mismo: "...la raíz es el órgano de las plantas que permite la fijación y la absorción de nutrientes y agua del suelo".

Las diferentes concepciones del término raíz se determinaron en las especies Encyclia cordigera, Manihot carthaginensis, Phoradendron mucronatum, Struthanthus dichotrianthus, Tillandsia flexuosa y Tillandsia recurvata localizadas en el bosque xerófilo y Avicennia germinans, Laguncularia racemosa y Rhizophora mangle pertenecientes al bosque de manglar, Tabla 1.

En algunas de las estaciones realizadas durante los recorridos al bosque xerófilo y de manglar, durante los trabajos de campo, los estudiantes recolectaron muestras botánicas de las raíces (sistema radical) de las especies vegetales en estudio, lo cual les permitió realizar observaciones y descripciones de las características físicas (anatómicas y morfológicas), así como de los rasgos sobre su interacción con el ambiente (ecológicos) y el papel metabólico que desempeñan en la planta (fisiológicos), Tabla 1.

\section{Concepción 1: raíces almacenadoras de sustancias de reserva}

Los estudiantes resultaron sorprendidos al observar los engrosamientos de las raíces del arbusto $M$. carthaginensis conocido como "yuca brava" y perteneciente a la familia Euphorbiaceae, destacando los rasgos morfológicos de alargamiento y de estructuras esférico-globosas, Tabla 1.

Estudiantes... "no sabíamos que existían raíces con modificaciones como esos abultamientos engrosados en su estructura".

Estudiantes..."se parece mucho a la yuca que se come, que también es una raíz".

Algunos estudiantes expusieron premisas referentes a la similitud de los rasgos físicos de la raíz de "yuca brava" como el color marrón de la cubierta ("concha"), su aspecto alargado y forma cilíndrica (imágenes visuales), además del olor que se apreció luego que fue cortada y desprendida la cubierta de dicha raíz (imagen olfativa), Tabla 2.

Las observaciones descritas resultaron clave para que la gran mayoría de los participantes asociaran y vincularan estas novedosas experiencias vivenciales a sus ideas previas y de la vida cotidiana de la raíz denominada yuca o mandioca de la especie Manihot esculetum, cultivada en la zona y empleada muy comúnmente como fuente de alimento para el consumo humano, Tabla 2. 
Tabla 1. Rasgos anatómicos, morfológicos, ecológicos y fisiológicos de las raíces de las especies identificadas durante el trabajo de campo

\begin{tabular}{|c|c|c|c|c|}
\hline $\begin{array}{l}\text { Especie vegetal/ } \\
\text { Rasgos radicales }\end{array}$ & Anatómicos & Morfológicos & Ecológicos & Fisiológicos \\
\hline Avicennia germinans & Neumatóforos & $\begin{array}{l}\text { Raíces cilíndricas } \\
\text { delgadas, con extremos } \\
\text { puntiagudos }\end{array}$ & $\begin{array}{l}\text { Interacción con el sedimento } \\
\text { anóxico o hipóxico }\end{array}$ & $\begin{array}{l}\text { Captación de oxígeno } \\
\text { atmosférico }\end{array}$ \\
\hline Encyclia cordigera & Raíces delgadas & Raíces delgadas & $\begin{array}{l}\text { Interacción epífita-sustrato } \\
\text { Epifitismo }\end{array}$ & $\begin{array}{l}\text { Fijación mecánica } \\
\text { (adherencia prensil) }\end{array}$ \\
\hline Laguncularia racemosa & Neumatóforos & $\begin{array}{l}\text { Raíces cilíndricas, con } \\
\text { extremos redondeados }\end{array}$ & $\begin{array}{l}\text { Interacción con el sedimento } \\
\text { anóxico o hipóxico }\end{array}$ & $\begin{array}{l}\text { Captación de oxígeno } \\
\text { atmosférico }\end{array}$ \\
\hline Manihot cartagenensis & Raíces engrosadas & $\begin{array}{l}\text { Raíces cilíndrico- } \\
\text { alargadas y engrosadas } \\
\text { (esférico-globosas) }\end{array}$ & Interacción con el clima & $\begin{array}{l}\text { Acumulación de } \\
\text { sustancias energéticas de } \\
\text { reserva (carbohidratos) }\end{array}$ \\
\hline Phoradendron mucronatum & Haustorio & $\begin{array}{l}\text { Raíces alargadas } \\
\text { delgadas y digitiformes }\end{array}$ & $\begin{array}{l}\text { Interacción hemiparásita- } \\
\text { hospedera } \\
\text { Hemiparasitismo }\end{array}$ & $\begin{array}{l}\text { Absorción de agua y } \\
\text { nutrientes }\end{array}$ \\
\hline Rhizophora mangle & Raíces fúlcreas ("zancos") & $\begin{array}{l}\text { Raíces cilíndrico- } \\
\text { alargadas y arqueadas }\end{array}$ & $\begin{array}{l}\text { Interacción con el sedimento } \\
\text { fangoso e inestable, el oleaje y } \\
\text { la alta concentración de sal }\end{array}$ & $\begin{array}{l}\text { Estabilidad y "anclaje" } \\
\text { (fijación mecánica) y } \\
\text { ultrafiltración de sal }\end{array}$ \\
\hline Struthanthus dichotrianthus & Haustorio & $\begin{array}{l}\text { Raíces alargadas, } \\
\text { delgadas y digitiformes }\end{array}$ & $\begin{array}{l}\text { Interacción hemiparásita- } \\
\text { hospedera. } \\
\text { Hemiparasitismo }\end{array}$ & $\begin{array}{l}\text { Absorción de agua y } \\
\text { nutrientes }\end{array}$ \\
\hline Tillandsia flexuosa & Raíces delgadas & Raíces delgadas & $\begin{array}{l}\text { Interacción epífita-sustrato } \\
\text { Epifitismo }\end{array}$ & $\begin{array}{l}\text { Fijación mecánica } \\
\text { (adherencia prensil) }\end{array}$ \\
\hline Tillandsia recurvata & Raíces delgadas & Raíces delgadas & $\begin{array}{l}\text { Interacción epífita-sustrato } \\
\text { Epifitismo }\end{array}$ & $\begin{array}{l}\text { Fijación mecánica } \\
\text { (adherencia prensil) }\end{array}$ \\
\hline
\end{tabular}


Tabla 2. Contribuciones didácticas de las concepciones del término raíz alcanzadas por los estudiantes durante el trabajo de campo

\begin{tabular}{|c|c|}
\hline Contribuciones & Alcances \\
\hline Brinda abundante información (observación olfativa, táctil y visual) & Focaliza la atención en "situaciones problema" (experiencias de aprendizaje) \\
\hline Proporciona las muestras botánicas como objetos de aprendizaje & Consolida el analisis y fortalece las conclusiones del aprendizaje \\
\hline Promueve la interacción ideas previas-experiencias de campo & Analiza y concluye sobre las nuevas interrelaciones cognitivas establecidas \\
\hline Favorece el uso del conocimiento científico disciplinar & Adquiere nuevo lenguaje científico y enriquece el conocimiento disciplinar \\
\hline Facilita los procesos mentales y el uso del método científico & Aplica los procesos mentales y el método científico en el nuevo aprendizaje \\
\hline Vincula el conocimiento botánico con otras disciplinas & Afianza las interacciones ecológicas y las adaptaciones al ambiente \\
\hline Estimula la motivación por los aprendizajes sorprendentes & Compara y analiza nuevas concepciones con el concepto tradicional de raíz \\
\hline Permite la formulación de ideas desde las experiencias vivenciales & Socializa el conocimiento sistematizado y científicamente aceptado \\
\hline
\end{tabular}


En relación a ello, Correa (2009) señaló sobre la importancia de la influencia del conocimiento previo de los educandos, el cual se organiza y se conecta con la información nueva, mediante la construcción de esquemas enraizados en las experiencias personales y culturales; destacando además que si este conocimiento, novedoso para los estudiantes, no se le presenta conectado con situaciones o ideas previas, se les hará difícil la construcción de nuevos esquemas mentales. En esto último radica justamente el valor sustancial que el docente le confiera al "morral" de experiencias cotidianas que los alumnos llevan a la clases.

Los alumnos además expresaron que las semejanzas entre las raíces de estas dos especies se podrían asociar a que ambas pertenecen al mismo género taxonómico Manihot y a la familia botánica Euphorbiaceae, lo que representó un nuevo afianzamiento cognitivo de la Sistemática y Taxonomía Vegetal. Lo señalado evidencia la fortaleza que ofrecen las experiencias de la vida cotidiana para vincularlas con el quehacer académico y el enriquecimiento del conocimiento científico disciplinar, para finalmente lograr consolidar el aprendizaje significativo, Tabla 2.

También los participantes evocaron conocimientos adquiridos en la cátedra Bioquímica, en la que aprendieron que la raíz yuca o mandioca ( $M$. esculetum) es rica en el polisacárido almidón (carbohidrato) y su consumo constituye una muy buena fuente de energía en la dieta el ser humano, Tabla 2. Esto último se asoció con las semejanzas en los caracteres físicomorfológicos (engrosamientos) de ambas raíces y que las mismas obedecen a cambios en la estructura de este órgano, dados justamente por la acumulación de sustancias de reserva energética (carbohidratos) que la planta emplea en su gasto metabólico.

La vinculación de la Botánica con el resto de las disciplinas permitó a los estudiantes a valorar la importancia de los aprendizajes previos y a concebir el conocimiento científico como una "red interconectada de saberes interdependientes", Tabla 2. En relación a ello, Vera y Martínez (2013) señalaron que estudiantes de la cátedra Ecología aplicaron sus conocimientos sobre fotosíntesis, adquiridos en la disciplina Fisiología Vegetal, al tema de productividad ecológica durante el trabajo de campo realizado al bosque de manglar.

\section{Concepción 2: raíces delgadas para la fijación mecánica}

Las raíces delgadas se presentaron en las especies Encyclia cordigera ("orquídea"), Tillandsia flexuosa y Tillandsia recurvata ("bromelias") de las familias Orquidiaceae y Bromeliaceae respectivamente, Tabla 1.

Estudiantes..."las plantas que viven sobre otras plantas se llaman epífitas".

Los estudiantes observaron que las raíces de estas tres especies de epífitas no las fijaban al suelo (observación visual), Tabla 2. A partir de discusiones intergrupales, los participantes concluyeron que tales estructuras radicales no cumplen la función de absorber agua y ni nutrientes.

Por otro lado, el uso y la manipulación de las muestras de estas plantas epífitas y sus raíces, les permitió a los alumnos desprender (separar) fragmentos de estas últimas que se encontraban estrechamente unidas al tallo o rama de la planta que le servían de sustrato de fijación y soporte, Tabla 2.

La situación vivencial de aprendizaje demostró que los estudiantes encontraron evidencia directa que las raíces de las plantas 
epífitas "no encajaba ni coincidían" con los rasgos y características del concepto tradicional de raíz, Tabla 2.

Ante experiencias como estas Vera et al., (2015) revelaron que los estudiantes al "toparse" con organismos que presentan rasgos anatómicos y estructurales (órgano) sobresalientes y que no coinciden con las ejemplificaciones tradicionales o referentes teóricos que comúnmente han observado o conocido, resultan sorprendidos ante tal acontecimiento. En dicha situación los aprendices deben estar conscientes e incorporar este nuevo aporte a su conocimiento como un "descubrimiento innovador" aunque el mismo no se corresponda con lo convencionalmente establecido en sus esquemas mentales previos.

La puesta en práctica de estas experiencias permitió que los estudiantes corroboraran que las raíces de las epífitas se extendían como prolongaciones delgadas adheridas a la superficie de la otra planta (tallo o rama) manteniéndolas sostenidas; de esta forma se concluyó que las mismas cumplen función de fijación mecánica.

Las raíces de las plantas epífitas permitieron además referir a los estudiantes, a través de la mediación de los docentes, que estos órganos permiten establecer una interacción ecológica plantaplanta correspondiente a un comensalismo conocido más específicamente como epifitismo. En esta interacción la planta epífita resulta beneficiada porque obtiene un sustrato físico donde adherirse que le brinda fijación mecánica mientras que la otra planta (tallo y/o rama), considerada como hospedera o de soporte, se mantiene inalterada.

En la experiencia antes descrita, se puso de manifiesto el valor agregado didáctico de los trabajos de campo realizados, ya que permitieron abordar obviamente tanto contenidos temáticos de Botánica como la oportunidad valiosa de interconectar con otros conocimientos disciplinares, destacando los de Ecología, Tabla 2.

\section{Concepción 3: raíces haustorios}

Los participantes se sintieron atraídos al encontrar otras plantas tipo arbustos cuyas raíces les permiten mantenerse unidas a las ramas de otras especies leñosas arbóreas. En primer lugar, los estudiantes establecieron comparación con las raíces delgadas de las epífitas, y luego pudieron diferenciarlas de estas últimas en que las raíces no estaban unidas ni revistiendo la superficie del sustrato; en lugar de ello el sistema radical penetraba a la parte interna de la rama y no era fácilmente removible, es decir, de separar o desprender, Tabla 2.

Estudiantes..."observamos sobre un árbol unas prolongaciones de una planta que parecían raíces penetrando en las ramas del árbol".

Estudiantes..."pensábamos que las raíces solo sostenían a las plantas al suelo".

Estudiantes..."no sabíamos que existían plantas que usaban las raíces para sacar agua y nutrientes de las ramas de otras plantas".

Este tipo de raíces, "descubiertas" por los estudiantes durante las actividades prácticas vivenciales de campo, resultó un conocimiento novedoso para ellos y a su vez algunos afirmaron que si conocían a estas plantas que "crecían" y se desarrollaban sobre árboles, Tabla 2.

Además algunos alumnos también afirmaron que estas plantas recibían el nombre común de "pajarito", "pajarilla" o "guate e' pajarito" y eran conocidas por ellos. Sin embargo, los participantes, en 
general, coincidían en que ignoraban como se mantenían unidas estas plantas arbustos sobre las ramas de los árboles, Tabla 2.

La participación del docente sirvió para orientar a los estudiantes y referirles que el sistema de fijación o sostén de estas plantas es a través de raíces especializadas llamadas haustorios, que son caraterísticas de las plantas hemiparásitas Struthanthus dichotrianthus ("pajarito") y Phoradendron mucronatum ("guate e' pajarito") agrupadas en las familias botánicas Loranthaceae y Viscaceae respectivamente, Tablas 1 y 2. Tales hemiparásitas se encontraron predominantemente en las ramas de los árboles hospederos Quadrella odoratissima ("olivo negro) y Pithecellobium dulce ("yacure") de las familias Capparaceae y Mimosaceae de forma respectiva, de acuerdo a lo reportado por Vera et al., (2014).

Los estudiantes, empleando muestras botánicas recolectadas in situ, describieron a las raíces haustorios como prolongaciones alargadas, delgadas y en algunos casos proyectadas de manera digitiformes sobre las ramas de algunos árboles y finalmente penetraban al interior de estas, Tabla 2. También los alumnos refirieron que en otras ramas los haustorios eran menos visibles y solo se apreciaban puntos de "inserción", de unión o de fijación de las hemiparásitas sobre las ramas; áreas en las cuales se destacaban abultamientos, engrosamientos y deformaciones del tejido leñoso de la rama, que producen daños físicos (anatómicosmorfológicos) y necrosis de la misma, Tabla 2.

Este trabajo practico, usando el material vegetal de las raíces haustorios, resultó muy importante ya que a los participantes les permitó discernir con mayor detalle sobre las propuestas, dudas, interrogantes e hipótesis planteadas, Tabla 2. A favor de estos hallazgos, Méndez Méndez y González (2019) han expuesto que las muestras biológicas promueven en los alumnos el contraste de la teoría con la práctica, la activación de saberes, las experiencias empíricas, la motivación para la construcción de conceptos y la integración de dimensiones actitudinales, procedimentales y conceptuales del conocimiento científico.

La experiencia vivencial le permitió a los estudiantes aprender sobre la interacción ecológica hemiparasitismo establecida entre la planta hemiparásita Struthanthus dichotrianthus (Loranthaceae) y la especie hospedera "olivo negro" Quadrella odoratissima (Capparaceae), Tabla 2.

Los participantes aprendieron que las plantas hemiparásitas poseen clorofila, son capaces de hacer actividad fotosintética, extraen agua y nutrientes de las plantas hospederas, y se conocen comúnmente como "pajarito". Los frutos de estas plantas son consumidos por aves que defecan las semillas sobre las ramas de los árboles en las que germinan, extienden sus raíces, penetran en las ramas, se desarrollan y crecen permaneciendo adheridas y tomando de agua y nutrientes de los conductos vasculares (xilema y floema). .

Durante la realización de los trabajos de campo se demostró el uso y manejo de una cantidad elevada de vocablos de terminología científico-técnica, también llamado lenguaje disciplinar (nomenclatura de códigos especializados).

En esta investigación, el lenguaje disciplinar botánico estuvo representado por un conjunto de códigos nominales como epítetos (nombres científicos de las plantas), familias botánicas, nombres específicos 
acuñados a raíces especializadas, interacciones ecológicas como las del tipo planta-planta entre otras; las cuales permitieron la comunicación y el entendimiento de la información generada y manejada, y favorecieron el aprendizaje y la adquisición de nuevos conocimientos por parte de los estudiantes durante la praxis didáctica, Tabla 2.

Los resultados mencionados anteriormente coinciden con Vera y Martínez (2013) quienes han señalado que los estudiantes, durante todo el desarrollo de las actividades de campo entran en contacto dinámico con la trilogía docente-ecosistemaotros participantes lo que les permite ganar valiosas experiencias de aprendizaje que incluyen el uso y manejo de un vocabulario disciplinar que incluye términos, procesos y aspectos metodológicos, entre otros elementos.

\section{Concepción 4: raíces fúlcreas}

Los estudiantes identificaron las raíces fúlcreas en Rhizophora mangle ("mangle rojo"), de la familia Rhizophoraceae en el bosque de manglar, las cuales describieron como prolongaciones alargadas, leñosas, arqueadas de 1 a 1,5 $\mathrm{m}$ de longitud que sobresalen y se proyectan por encima de la superficie del agua, se unen al tallo de los árboles mangle rojo y lo mantienen firmemente "anclado" al sedimento fangoso, Tabla 1.

Estudiantes..."los mangles poseen raíces zancos, arqueadas y expuestas a la superficie que le permiten fijarse a terrenos blandos (tierras húmedas)".

Estudiantes... "nos encantó el mangle rojo, es un árbol de gran tamaño y resultó sorprendente observar como sus raíces son tan grandes y sobresalen".
La experiencia de los alumnos con las raíces fúlcreas del mangle rojo resultó didácticamente muy enriquecedora. Inicialmente la imagen (observación visual) de los rasgos anatómicos y morfológicos de estos órganos vegetales causó un impactó sorprendente en los participantes, Tabla 2.

Por otra parte, los estudiantes desarrollaron actividades didácticas como la realización de observaciones de imágenes táctiles importantes dado que tocaron y removieron las raíces fúlcreas del mangle rojo y el desplazamiento o caminata sobre el suelo o sedimento "flojo", fangoso, pantanoso del manglar, Tabla 2.

Estas experiencias in situ condujeron al planteamiento de ideas, supuestos y a la confirmación de una interrelación plantasuelo: raíces fúlcreas grandes y vigorosas que dan "anclaje" físico-mecánico en un sustrato poco firme $e$ inestable, $y$ concluyendo además que se trata de una adaptación de la especie al ecosistema, Tabla 2. De igual forma, los docentes en su rol mediador, reforzaron conocimientos a los estudiantes haciendo señalamientos en que estas raíces del mangle rojo también cumplen la función de ultrafiltración de las sales; regulando de esta manera el ingreso de estos compuestos a la planta.

En relación a estos hallazgos, Acosta ef al., (2017) han afirmado que los trabajos de campo promueven en los estudiantes la activación de los sentidos, la participación grupal y los procesos de recolección de información, interpretación, formulación de hipótesis y experimentación; fomentado de esta forma adquisición de competencias científicas.

Igualmente Vera y Martínez (2013) señalaron que las actividades de campo en las ciencias naturales brindan a los alumnos 
la oportunidad de afianzar, agudizar e incrementar su capacidad de observación, dado que el entorno le ofrece una gran cantidad de información a manera de experiencias en su mayoría visuales, sin dejar a un lado las auditivas, gustativas, olfativas, y táctiles.

\section{Concepción 5: raíces neumatóforos}

Las raíces neumatóforos se encontraron en las especies Avicennia germinans (mangle negro) y Laguncularia racemosa (mangle blanco) de las familias Avicenniaceae y Combretaceae respectivamente, en el bosque de manglar, Tabla 1.

Estudiantes..."observamos directamente palitos que salían de la superficie del suelo fangoso e inundado del manglar que parecían plantas pequeñas sin hojas".

Estudiantes..."tocamos estos pequeños tallos y tratamos de arrancarlos pero estaban fuertemente unidos al suelo, se rompían y no presentaban raíces".

La motivación de los participantes se enfocó en este tipo de órganos radicales dado que, a través de la observación visual, éstos tuvieron la "inquietud o curiosidad" por estas estructuras leñosas delgadas emergentes del sedimento y localizadas al pie de los árboles de Avicennia germinans y Laguncularia racemosa, Tabla 2.

Al igual que en las experiencias vivenciales previas, alcanzadas en el trabajo de campo, los estudiantes expresaron durante las sesiones orales (mini plenarias) la información recabada de las estructuras anatómicas encontradas, producto de:

1) La observación visual y táctil, la cual consistió en la fijación de imágenes captadas in situ a través del sentido de la vista, como la disposición vertical, color marrón y emergencia del suelo; y por medio del tacto al tocar tales estructuras, captar su textura, corroborar la firmeza con que estaban unidas al sustrato y lo difícil que resultó desprenderlas del mismo, Tabla 2.

2) La descripción de los rasgos físicos y morfoanatómicos de estos órganos, permitió exponer y detallar: la naturaleza leñosa, la forma alargada, la estructura cilíndrica delgada $\circ$ gruesa y los extremos redondeados o puntiagudos, Tabla 2 .

Además, en esta experiencia es importante destacar que los participantes lograron identificar a los neumatóforos del mangle blanco y a los neumatóforos del mangle negro, Tabla 2.

Para ello los estudiantes hicieron uso de la comparación (establecimiento de diferencias y semejanzas), clasificación de estas estructuras hasta finalmente concluir que los neumatóforos se podían diferenciar según la especie de mangle a la que pertenecen (criterio de especificidad) y atendiendo justamente a los rasgos físicos y morfoanatómicos señalados previamente: Avicennia germinans (mangle negro) presentó neumatóforos delgados de extremos puntiagudos y Laguncularia racemosa (mangle blanco) exhibió neumatóforos gruesos de extremos redondeados, Tabla 2.

En todas las experiencias señaladas los alumnos aplicaron los procesos mentales y el método científico hasta obtener elementos válidos y concluyentes en los nuevos aprendizajes alcanzados, Tabla 2. Esta afirmación coincide con el reporte de Arteaga y Tapia (2009) quienes han indicado que el iqué enseñar?, no solo debe contemplar aspectos conceptuales, sino que además incluye habilidades, y el desarrollo de la actividad creadora e investigativa.

3) La comparación, conexión con las semejanzas y diferencias de otras estructuras 
vegetales, permitió asociar esta nueva información sobre neumatóforos con los aprendizajes adquiridos sobre plantulas, pequeños tallos y raíces, los cuales fungieron como ideas previas tanto de la vida cotidiana como del conocimiento científico académico, Tabla 2. Esto sirvió para descartar algunas premisas y finalmente concluir que se trataban de raíces especializadas denominadas neumatóforos.

4) El aspecto motivación también se manifestó entre los estudiantes, dada que esta experiencia de aprendizaje despertó el interés y la inquietud entre ellos al "conocer" que los neumatóforos se desarrollaban ("crecían") en dirección contraria a la fijación al sustrato, es decir, con geotropismo negativo; lo cual contradice lo establecido en la concepción tradicional del término raíz manejada por gran parte de los estudiantes, Tabla 2.

La situación de aprendizaje con los neumatóforos se constituyó en un "reto", aunado a la curiosidad despertada en los estudiantes, que afianzó el interés y la motivación para descifrar la incógnita sobre tales estructuras y cuyo interés se originó a partir de la vivencia empírica del campo, y sobre la cual algunos aprendices ya conocían aspectos sobre los manglares producto de la vida cotidiana, Tabla 2.

Sin embargo, Arteaga et al., (2012) han señalado que comúnmente existe falta de motivación de los estudiantes por los contenidos curriculares de Biología. A objeto de mejorar esta situación Rodríguez Moneo (2009) propone indagar y establecer las conexiones entre los planteamientos de la Biología, y la Botánica en particular, y las vivencias cotidianas de los alumnos a fin de elevar el interés por el aprendizaje de los contenidos de estas disciplinas.
Estudiantes..."nunca pensamos que existían raíces que crecían hacia arriba en dirección contraria al suelo".

La premisa anterior revela la existencia de un hallazgo novedoso y sin precedentes que inicialmente generó tropiezos con la concepción convencional del término raíz de los participantes ("choque cognitivo") y a partir de esta situación se produjo la aceptación de una nueva percepción conceptual, Tabla 2.

En relación a esta afirmación Siqueira y Porlán (1997), con base en la teoría epistemológica de Toulmin, expresaron que el aspecto racional de las actividades intelectuales se asocia a la manera con que cada persona es capaz de modificar su posición intelectual (microrrevoluciones o unidades de variación) frente a experiencias nuevas e imprevistas.

Ante esta experiencia de aprendizaje, el cambio conceptual considera a los hechos empíricos con el objeto de construir una representación, nomenclaturas y procedimientos explicativos mejores, es decir, se parte de la experiencia propia vivencial del individuo la cual se toma como plataforma para la elaboración y confección de constructos cognitivos más elaborados y por ende complejos (Siqueira y Porlán, 1997).

5) La experiencia vivencial promovió el análisis y la confirmación de la interacción ecológica planta-atmósfera, dado que los neumatóforos cumplen con la función de tomar oxígeno atmosférico para la respiración de la planta, Tabla 2. Dicha función metabólica también se asoció a las condiciones de hipoxia o anoxia que caracterizan al sedimento del manglar y la misma obedece a una adaptación de las especies de mangle al ecosistema, Tabla 2. 
Las situaciones de aprendizaje alcanzadas durante las vivencias de los trabajos campo resultaron muy importantes y altamente enriquecedoras.

Las actividades además de ofrecer grandes aportes didácticos al fortalecimiento de la formación académica de los estudiantes, también brindaron la oportunidad para que éstos confrontaran sus ideas organizadas y los saberes alcanzados, los divulgaran con el resto de los alumnos y finalmente fueran compartidos como conocimientos y saberes sistematizados y aceptados dentro del marco de la ciencia, Tabla 2.

Todo esto encierra un gran aporte educativo para los participantes, lo cual coincide con Tobar et el., (2020) quienes señalaron que los estudiantes en el manejo de la huerta escolar desarrollaron competencias tales como la capacidad de análisis e interpretación de lo observado, el fortalecimiento de la convivencia, la tolerancia y la aceptación de las ideas contrarias a las propias a partir del trabajo en equipo, el enriquecimiento del proceso de aprendizaje por medio de la interacción entre compañeros y la aplicación de las fases del método científico que los motivó a realizar un trabajo riguroso y responsable.

CONCLUSIONES

Las diferentes concepciones del término raíz resultaron novedosas para los estudiantes, y las experiencias de aprendizaje adquiridas podrían generar cambios conceptuales en sus estructuras cognitivas para enriquecer el conocimiento sobre el mismo.

\section{REFERENCIAS}

Acosta, S., Fuenmayor, A. y Sánchez, A. (2017). El trabajo de campo como estrategia didáctica para el aprendizaje de la Zoología. Omnia, 23(1), 59-78

Acevedo, J. (2004). Reflexiones sobre las finalidades de la enseñanza de las ciencias: educación científica para la ciudadanía. Revista Eureka sobre Enseñanza y Divulgación de las Ciencias, 1(1), 3-16

Álvarez, S. (2007). ¿Cómo desean trabajar los alumnos en el laboratorio de Biología?. Un acercamiento a las propuestas didácticas actuales. Revista Iberoamericana de Educación, 42(7), 1 13

Arteaga, Y., Méndez, E. y Tapia, F. (2012). Núcleos problemáticos en el aprendizaje de la Biología. Multiciencias, 12(№ Extraordinario), 283-287

Arteaga, Y. y Tapia, F. (2009). Núcleos problemáticos en la enseñanza de la Biología. EDUCARE, 12(46), 719-724

Buitrago Suárez, N. (2011). El humedal madre vieja, un laboratorio para el aprendizaje significativo y el desarrollo de habilidades de pensamiento. Trabajo de grado presentado como requisito parcial para optar por el título de Magister en Enseñanza de las Ciencias Exactas y Naturales

Correa, A. (2009). ¿ Cómo hacer del aprendizaje de la Biología un asunto relevante para los estudiantes? Biografía: escritos sobre la Biología y su enseñanza, 2(3), 140-147

Méndez Méndez, E. y González, R. (2019). Las muestras biológicas: un análisis de su intencionalidad didáctica. Revista Paradigma, $\mathrm{XL}(1), 15-27$

Moreno, E. (2007a). La flora de los supermercados: un recurso para aprender taxonomía vegetal y algo más a través del enfoque CTS. XVII Congreso Venezolano de Botánica. Maracaibo, Venezuela. Consultado 10 de diciembre de 2019. Disponible en: http://www. docstoc.com/docs/13073944/La-florade-los-supermercados-un-recurso-para- 
aprender-taxonomia-vegetal-y-algomas-atraves-del-enfoque-CTS

Moreno, E. (2007b). El herbario como recurso para el aprendizaje de la Botánica. Acta Bot. Venez., 30(1), 415427

Oviedo, R. D. y Dávila, B. (2001). La enseñanza de la ecología en el patio de la escuela. Memorias de las $\mathrm{V}$ Jornadas Nacionales de Enseñanza de la Biología, La educación en Biología: tendencias e innovaciones. Misiones, Argentina 480-481

Padrón, J. (2016). Teoría de la investigación. Una visión evolucionista y cognitiva de la producción de investigaciones. DVD del Seminario Teoría de la investigación, Doctorado en Ciencias Humanas, Universidad del Zulia

Parisca, L., Lindorf, H. y Rodríguez, P. (1985). Botánica: clasificación, estructura y reproducción. Ediciones de la Biblioteca (Universidad Central de Venezuela). Caracas. Venezuela

Rodríguez Moneo, M. (2009). Motivar para aprender en situaciones académicas. En G. Romero y A. Caballero (eds.), La crisis de la escuela educadora. Barcelona: Laertes

Siqueira, J. B. y Porlán, R. (1997). La epistemología evolucionista de Stephen
Toulmin y la enseñanza de las ciencias. Investigación en la Escuela, 39, 17-26

Tacca, D. (2010). La enseñanza de las ciencias naturales en la educación básica. Investigación Educativa, 14(26), 139-152

Tobar, D. N., Carabalí-Banguero, D. J. y Bonilla, D. S. (2020). La huerta escolar como estrategia en el desarrollo de competencias y el pensamiento científico. Revista Interamericana de Investigación, Educación y Pedagogía, 13(1), 101-112

Vera, A., Fernández, O. y Bravo de Nava, E. (2015). Teoría evolucionista de Toulmin: fundamento epistemológico para el aprendizaje de la Ecología. Multiciencias, 15(2), 156-162

Vera, A. y Martínez, M. (2013). Bosque de manglar: ambiente para la enseñanza y aprendizaje de la Ecología. Multiciencias, 13(1), 46-52

Vera, A., Martínez, M., Parra, Y., Maldonado, R. y Carvajal, N. (2014). Plantas hemiparásitas y hospederas presentes en el bosque xerófilo de la Ciénaga de La Palmita, estado Zulia, Venezuela. Rev. Fac. Agron. (LUZ), Supl. $1,213-223$ 